\title{
Funcionamento cognitivo e habilidades metalinguísticas na aprendizagem da leitura
}

\section{Funcionamiento cognitivo $y$ habilidades metalingüísticas con el aprendizaje de la lectura}

\section{Cognitive operation and metalinguistic abilities in reading learning}

\author{
Sebastián Urquijo ${ }^{1}$
}

\begin{abstract}
RESUMEN
El objetivo de este trabajo es el de analizar las relaciones del funcionamiento cognitivo y el dominio de las habilidades metalingüísticas con el aprendizaje formal de la lectura durante el primer año de la escuela. Las habilidades metalingüísticas implican la reflexión conciente sobre los diferentes niveles del sistema lingüístico y se desarrollan durante la infancia, perfeccionándose con la escolaridad. Por otra parte, el funcionamiento cognitivo involucra procesos necesarios para percibir, seleccionar, procesar y almacenar información, tareas de relevancia en un proceso complejo como la lectura. Se evaluaron 127 niños y niñas, alumnos del primer año del ciclo básico de la educación primaria de dos escuelas públicas y dos privadas de la ciudad de Mar del Plata, Argentina, a quienes se les administraron instrumentos para determinar sus desempeños en memoria, atención, funciones ejecutivas, razonamiento, habilidades metalingüísticas y lectura. Los resultados indican, de forma sistemática, que tanto los procesos cognitivos como las habilidades metalingüísticas se asocian de manera significativa al desempeño en diferentes medidas de lectura. Las conclusiones discuten estas relaciones y advierten sobre la influencia de los factores individuales en el proceso de aprendizaje de la lectura.

Palabras-clave: procesos cognitivos; habilidades metalingüísticas; lectura; aprendizaje.
\end{abstract}

1 Doutor em Educação (Psicologia Educacional) - CONICET - Centro de Investigación en Procesos Básicos, Metodología y Educación (CIMEPB) - Facultad de Psicología - Universidad Nacional de Mar del Plata - Argentina - urquijo@mdp.edu.ar. Financiado por CONICET e UNMP. 


\title{
RESUMO
}

O objeto do trabalho é analisar as relações do funcionamento cognitivo e o domínio das habilidades metalinguísticas com a aprendizagem formal da leitura no primeiro ano do primeiro grau da escola básica. As habilidades metalinguísticas têm a ver com a reflexão consciente acima dos diferentes patamares do sistema linguístico e se desenvolvem na infância, aprimorandose com a escolaridade. Pela sua parte, o funcionamento cognitivo envolve processos necessários para perceber, selecionar, processar e armazenar informação, tarefas de importância num complexo processo como a leitura. Foram avaliados 127 meninos e meninas, alunos do primeiro ano, do primeiro grau de duas escolas da rede municipal de ensino e duas escolas particulares da cidade de Mar del Plata, Argentina, a quem lhes foram administrados instrumentos para avaliar seus desempenhos em memória, atenção, funções executivas, raciocínio, habilidades metalinguísticas e leitura. Os resultados indicam, de forma sistemática, que tanto os processos cognitivos como as habilidades metalinguísticas associam-se de forma significativa ao desempenho em diferentes medidas de leitura. As conclusões discutem essas relações e destacam a influência dos fatores individuais no processo de aprendizagem da leitura.

Palavras-chave: Processos cognitivos; habilidades metalinguísticas; leitura; aprendizagem.

\begin{abstract}
This work aims at analyzing the cognitive operation relations and the dominion of the metalinguistic abilities with the formal reading learning process during the school's first year. The metalinguistic abilities imply the conscientious reflection upon the different levels from the linguistic system and they are developed during childhood, perfecting itself with the schooling process. The cognitive operation involves necessary processes to perceive, select, process and store information, which are relevant tasks in a complex process like reading. A total of 127 primary education students from the Basic cycle first year of two public and two private schools of Mar del Plata city, Argentina, were evaluated with psychological instruments to determine their performances in memory, awareness, executive functions, reasoning, metalinguistic abilities and reading. The results indicate that both the mental processes and the metalinguistic abilities are associated significantly to the performance in different reading measures. The conclusions discuss these relations and highlight the individual factors influence in the process of reading learning.
\end{abstract}

Keywords: Cognitive processes; metalinguistic abilities; reading; learning. 
La adquisición de la lectura es un proceso complejo que descansa en el desarrollo de diversas funciones cognitivas (GOUGH, 2002; GOUGH; COSKY, 1975; JUST; CARPENTER; WOOLEY, 1982). Según el momento lector, el desarrollo cerebral, el método de enseñanza, el tipo de texto y las características del ambiente, se privilegia el uso de diversas estrategias, tales como la decodificación, el reconocimiento visual, el reconocimiento globalsemántico etc. Diversos estudios han mostrado que la eficiencia en la lectura se relaciona con la capacidad para decodificar estímulos visuales, la velocidad en la denominación, la amplitud de vocabulario, la capacidad de la memoria operativa y la habilidad para mantener la atención y concentración; especialmente, se ha encontrado una relación con las habilidades fonológicas y la conciencia fonológica en particular. La lectura requiere un sistema de análisis indirecto de la palabra que puede darse mediante el reconocimiento de cada uno de sus elementos no significativos (análisis sublexical) o del reconocimiento de la palabra en su totalidad (análisis lexical). Este modelo propone la existencia de una doble ruta en la lectura: una de ellas sería la ruta fonémica y la otra la lexicalsemántica (COLTHEART, 1981). De acuerdo con Ardila (1998), en español la lectura se lleva a cabo preferentemente mediante la identificación de grafemas y sílabas, y, por lo tanto, en español la ruta más importante sería la ruta sublexical o fonológica. El español tiene reglas de correspondencia grafema-fonema claras (MATUTE; LEAL, 2003), que no se observan en inglés.

La adquisición de la lectura se entiende como un proceso que tendría prerrequisitos cognitivos mediados por distintas estructuras cerebrales. Los prerrequisitos que con mayor frecuencia se han comunicado son el procesamiento fonológico, la denominación automatizada rápida, la automaticidad motora, la percepción del habla y la memoria a corto plazo (SAVAGE et al., 2005). Para Rosselli et al. (2006), la lectura exige, además, una serie de habilidades de tipo cognitivo, como son atención, memoria, lenguaje y abstracción. La atención es indispensable para lograr una adecuada decodificación de los estímulos y comprensión del texto. Los predictores cognitivos de la lectura varían, sin embargo, según el desempeño lector (SAVAGE et al., 2005; CANETJURIC; URQUIJO; RICHARD'S; BURIN, 2009). Existen investigaciones que vinculan de forma directa a las funciones ejecutivas y la adquisición de la lectura (ALTEMEIER et al., 2006; WILLCUTT et al., 2005; SWANSON, 1999). Por otra parte, existen estudios que relativizan esas relaciones (PURVIS; TANNOCK, 1997; SENGSTOCK, 2001; RUMSEY, 2004; LEE et al., 2004; STRINGER; TOPLAK; STANOVICH, 2004). Además de los prerrequisitos cognitivos, el aprendizaje de la lectura se asocia a numerosos factores ambientales, tales como la exposición a la lectura, al contexto alfabetizador, o al desarrollo de una actitud y motivación positivas. Leer y escribir constituyen 
esencialmente prácticas sociales y comunicativas que implican el desarrollo de las habilidades discursivas necesarias para alcanzar determinados propósitos (MOJE; DILLON; O'BRIEN, 2000; GEE, 1999; LEE, 1995; HOURIGAN, 1994; STREET, 1984; SCRIBNER; COLE, 1981; ANDRÉS; URQUIJO; NAVARRO; GARCIA-SEDEÑO, 2010). Los niños que viven en ambientes socioculturales pobres tienden a tener un desempeño lector limitado y mayores dificultades en el aprendizaje de la lectura (URQUIJO, 2009, WIGFIELD; ASHER, 2002).

Las habilidades metalingüísticas se refieren a la reflexión conciente sobre los diferentes niveles del sistema lingüístico (MORASO; DURO, 2004), es decir que cada nivel que compone el lenguaje puede ser tomado como objeto de reflexión. Cada uno de estos niveles alude a distintos tipos de habilidades metalingüísticas (BORZONE; ROSEMBERG; DIUK; SILVESTRI; PLANA, 2004). La conciencia fonológica se refiere a la toma de conciencia sobre los sonidos de la propia lengua (DEFIOR CITOLER, 1994). La conciencia léxica implica la capacidad de aislar las palabras que componen una oración y comprender que se trata de una unidad lingüística separada (BORZONE et al., 2004; CHÁVEZ ZAMORA, 2006). Esas habilidades se van perfeccionando en los niños, alcanzando grados mayores de experticia con el aprendizaje de la lectura (BORZONE et al., 2004). De acuerdo con Ball (1997), Ott (1997) y Stiller (2005), la mejoría en esas habilidades está relacionada con la escolarización. De todas maneras, su desarrollo progresivo es un tema complejo que requiere que se realicen más estudios longitudinales que permitan esclarecer cómo es la adquisición paulatina de esas capacidades (GUIMARÃES, 2002). La revisión de la literatura científica revela la existencia de escasos estudios con niños hispanoparlantes (DEFIOR CITOLER, 1994).

Actualmente, el concepto de "desarrollo" se entiende de forma diferente, eliminando dicotomías como la de "naturaleza y ambiente". Según Catherwood (2000), tampoco se sostienen ideas que suponen su inicio desde el nacimiento, a partir de reflejos; negar la multimodalidad del desarrollo o su separación del aprendizaje. Hoy, la idea de desarrollo

[...] responde a un modelo integrado en el que la ontogenia se entiende como una organización jerárquica sucesiva y ordenadamente emergente, con relaciones bidireccionales entre la complejidad biológica y la organización psicológica, incluyendo la actividad genética y la neurológica, la experiencia y el entorno [...] (SASTRE RIBA, 2006, p. 144). 
La estructura neural influye en las funciones psicológicas y el aprendizaje, y éstos, en aquélla. Entonces, el desarrollo cognitivo se entiende como la transformación continua y diferencial de estructuras y funciones cognitivas durante el ciclo vital, a partir de conductas preformadas y en interacción con el medio. En ese proceso, la acción individual y la interacción social son componentes esenciales (HAAN; JONSON, 2003); el interés reside no sólo en las capacidades cognitivas construidas y reconstruidas, sino también en su control, funcionamiento y manifestaciones diferenciales. A lo largo de este proceso, la organización y el despliegue funcional de conductas van dando lugar a cursos de desarrollo armónicos o disarmónicos. La estructura cerebral, el desarrollo cognitivo y el aprendizaje están estrechamente imbricados en la sucesiva construcción intelectual y en sus manifestaciones. Esa transformación genera manifestaciones diferenciales que pueden cursar hacia la deficienciación del sujeto o hacia dificultades en el aprendizaje escolar o social. De ahí la importancia de conocer la organización y el despliegue funcional de conductas que van dando lugar, sucesivamente, al estado cognitivo y a las posibilidades de aprendizaje de un sujeto.

El desarrollo cognitivo se inicia a partir del sexto mes de gestación y está constituido por la puesta a punto de funciones como la atención, la percepción, la memoria, la imitación, la lógica y las funciones ejecutivas (GOSWAMI, 1998; MEHLER; DUPOUX, 1992), así como por diversos dominios de conocimiento: numérico, lingüístico, físico, biológico y psicológico. Sin embargo, muchas habilidades cognitivas se desarrollan lentamente y no alcanzan su pico hasta la edad adulta (DELUCA et al., 2003; DIAMOND, 2002; LUCIANA; NELSON, 2002; LUCIANA et al., 2005; LUNA; GARVER; URBAN; LAZAR; SWEENEY, 2004; LYONS-WARREN; LILLIE; HERSHEY, 2004; MUNOZ; BROUGHTON; GOLDRING; ARMSTRONG, 1998; ZELAZO; CRAIK; BOOTH, 2004).

El desarrollo cognitivo se posibilita funcionalmente mediante competencias preformadas, como la lógica (protológica), las funciones ejecutivas y la interacción social. La protológica permite, desde el inicio de la vida, la organización de la acción sobre el entorno y la información significativa. La eficacia de la actividad lógica y de sus productos reclama la pertinencia del funcionamiento ejecutivo en la planificación, control y flexibilidad en la génesis y aplicación de los esquemas de resolución. Sus componentes más destacados son tres (ANDERSON; DOYLE, 2003; ZELAZO, 2004; SHALLICE, 2001): el Control de la atención (atención selectiva, sostenida e inhibición); el Establecimiento de un objetivo (iniciativa, planificación, organización y estrategias de resolución) y la Flexibilidad cognitiva (memoria de trabajo, cambio atencional, monitoreo, transferencia y autorregulación). 
La investigación actual sobre desarrollo cognitivo concede alta relevancia al papel de las funciones ejecutivas y la atención. Se consideran básicamente un constructo que comprende habilidades centrales autorreguladoras, que orquestan procesos de dominio específico con el fin de lograr un objetivo flexiblemente (ELLIOTT, 2003). Cumplen funciones de control, supervisión o autorregulación que organizan toda la actividad cognitiva y la emocional. Las más estudiadas son la inhibición de respuestas dominantes, la planificación y monitorización y el shifting o conmutación, todas ellas relacionadas con la flexibilidad cognitiva (DEAK; NARASIMHAM, 2003) o con la perseveración e impulsividad (AKSAN; KOCHANSKA, 2004).

El funcionamiento cognitivo está facilitado por el control ejecutivo que coordina la consecución de un objetivo, la flexibilidad y la regulación cognitiva. Facilitan la toma de decisiones (intencionalidad), la selección y conservación de la información (representación), y la organización lógica y planificación de la acción. Sus componentes fundamentales son filtrar la información irrelevante (supresión de interferencias) e inhibir respuestas predominantes o no adecuadas (inhibición) que enmascaran o dificultan la competencia del niño. Las funciones ejecutivas cambian con la edad, son decisivas en cuanto al rendimiento social y académico, y tienen expresiones diferenciales según los cursos de desarrollo típico o atípico, respecto a los que explican aspectos importantes. El déficit en algunos aspectos condiciona ciertos trastornos, de ahí la importancia de conocer sus raíces e identificar los mecanismos cognitivos subyacentes, como la incapacidad de resistir a la interferencia o la impulsividad. Esas disfunciones no están prefijadas, sino que son la expresión del sucesivo proceso de desarrollo. Pero no sólo la natural da lugar al desarrollo cognitivo, la interacción social trasciende y modula el funcionamiento neurobiológico individual; es decir, el desarrollo cognitivo es individual y social.

La influencia del desarrollo cognitivo, para explicar dificultades de aprendizaje en la edad preescolar y escolar, está demostrada (FEINSTEIN; BYNNER, 2004; MELLIER; FESSARD, 1998; SOMMERFELT et al., 2000). Un estudio de Etchepareborda y Abad-Mas (2005) indica que su afectación provoca una disfunción que influirá en procesos de aprendizaje formal académico: manejo de la atención, inhibición de estímulos irrelevantes, reconocimiento de patrones, reconocimiento de jerarquías y significado de los estímulos, formulación de intenciones, reconocimiento y selección de metas; establecimiento de un plan, análisis de actividades y dificultades para la ejecución de un plan, monitorización o modificación de la tarea. Todo ello redunda en la necesidad de investigar y comprender el desarrollo y el funcionamiento cognitivo y sus relaciones con el aprendizaje para conocer su soporte estructural y su funcionalidad. 
Las funciones ejecutivas se definen como un conjunto de habilidades cognitivas cuyo objetivo es la adaptación del individuo a situaciones nuevas y complejas (COLLETTE, HOGGE, SALMON; VAN DER LINDEN, 2006). Si bien aún no existe un acuerdo definitivo sobre cuáles son estos subprocesos, se suele incluir entre ellos a la inhibición (DELIS; KAPLAN; KRAMER, 2001; DENCKLA, 1994), el control atencional (ANDERSON, 2001), la flexibilidad cognitiva (DENCKLA, 1994; LAFLECHE; ALBERT, 1995; ANDERSON, 2001; PIGUET; GRAYSON; BROWE et al., 2002) y la memoria de trabajo (PENNINGTON; OZONOF, 1996).

Con respecto a la inhibición, se trata de la habilidad para controlar información irrelevante durante la realización de una actividad. Para que el individuo pueda hacer una selección apropiada de la información pertinente y mantener su atención durante periodos prolongados, es esencial que aprenda a inhibir respuestas que surgen de manera automática (WODKA; MAHONE; BLANKNER et al., 2007).

Según Papazian, Alfonso y Luzondo (2006), la flexibilidad cognitiva consiste en un proceso mental que depende de la edad, cuya capacidad limitada para cambiar intermitentemente de una a varias reglas o estrategias impone demandas adicionales a los procesos de inhibición y a la memoria de trabajo. En una tarea que requiere flexibilidad cognitiva, el foco de la atención debe ser desplazado de una clase de estímulo a otra, y el sistema de control debe permitir alternar entre dos sets cognitivos diferentes. Esa habilidad implica un análisis de las consecuencias de la propia conducta y un aprendizaje de los errores. La flexibilidad depende del número de reglas incluido dentro de la tarea, por eso, al incrementar el número de reglas y la complejidad de la tarea, los niños producen mayor número de respuestas perseverativas que denotan menor flexibilidad.

De acuerdo con el modelo de Baddeley (1992, 1996, 1998; BADDELEY; HITCH, 1994; BADDELEY; DELLA SALA, 1996), la memoria de trabajo incluye una estructura llamada ejecutivo central, considerada responsable del control y de la regulación de los procesos cognitivos. A su vez, cuenta con dos sistemas subordinados: el bucle o lazo articulatorio, que transforma estímulos verbales auditivos y visuales en un formato fonológico, y que incluye un almacenamiento pasivo y un proceso de repaso que permite refrescar la información evitando el decaimiento de la huella mnémica (BADDELEY; LARSEN, 2007); el otro subsistema es la agenda viso-espacial, que se encarga del procesamiento y almacenamiento visual y espacial de imágenes mentales. Goldman-Rakic (1996), Roberts y Pennington (1996) señalan que la memoria de trabajo consiste en un proceso on-line de capacidad limitada que sostiene una representación de la información en un breve período de tiempo, luego la procesa en un dominio computacional (ej. aritmética mental) y la asocia con 
otras ideas y con información entrante, facilitando la elección de una respuesta para alcanzar un objetivo.

Las investigaciones que han abordado las funciones ejecutivas y las habilidades metalingüísticas han privilegiado la evaluación de la conciencia fonológica y se han centrado mayormente en niños con déficit de atención con y sin hiperactividad (VAQUERIZO-MADRID; ESTÉVEZ-DÍAZ; DÍAZMAÍLLO, 2006; BARCELÓ MARTÍNEZ; LEWIS HARB; MORENO TORRES, 2006; ROMERO-AYUSO; MAESTÚ; GONZÁLEZ-MARQUÉS; ROMOBARRIENTOS; ANDRADE, 2006), sin explicitar cuál es la relación que se establece entre las funciones ejecutivas y la reflexión sobre los componentes del lenguaje. Podría esperarse que un mayor desarrollo de las funciones ejecutivas, que implican el control deliberado de una serie de alternativas en el plano del pensamiento, se relacione positivamente con un mejor uso de las habilidades metacognitivas de naturaleza metalingüística, que implican tomar un objeto -lingüístico en este caso - y reflexionar conscientemente sobre él.

La relación positiva entre funciones ejecutivas y habilidades metalingüísticas podría explicarse por el componente metacognitivo, en tanto éste supone un monitoreo activo y una regulación y orquestación del procesamiento de la información sobre un objeto de reflexión que posee una meta cognitiva (FLAVELL, 1976). La relación de la lectura con los procesos mnésicos resulta obvia en la medida en que aprender consiste en almacenar y recuperar información.

Sin embargo, y a pesar de los numerosos estudios, la relación entre las funciones ejecutivas, la atención, la memoria, el razonamiento, las habilidades metalingüísticas y la lectura resulta controversial. El análisis de las variables cognitivas asociadas con las habilidades de lectura permite incrementar la comprensión, no sólo de los factores subyacentes a la lectura exitosa, sino también de los problemas en su aprendizaje, particularmente, el estudio de las demandas cognitivas de las funciones ejecutivas que exige la adquisición de la lectura. Por todo lo expuesto, este trabajo se propone a explorar y describir las relaciones entre las funciones ejecutivas, la atención, la memoria, el razonamiento, las habilidades metalingüísticas y la lectura.

\section{Metodología}

\section{Sujetos}

Se trabajó con una muestra definitiva de 127 niños y niñas, alumnos del primer año del ciclo básico de la educación primaria, de escuelas públicas y 
privadas de la ciudad de Mar del Plata, Argentina. Se obtuvieron datos de 61 alumnos de dos escuelas públicas escogidas al azar entre las 17 escuelas de la Secretaría de Educación de la Municipalidad de General Pueyrredón y de 66 alumnos de dos escuelas privadas seleccionadas de manera intencional, una de ellas bilingüe, con doble escolaridad y otra con escolaridad simple. En cada escuela se seleccionaron al azar los cursos y se evaluó a todos los alumnos cuyos padres hubieran prestado el consentimiento para la prueba. Durante el período de evaluación, algunos de los niños no asistieron a la escuela y otros no completaron correctamente las pruebas. Se excluyeron de la muestra niños y niñas repetidores y con problemas o trastornos específicos del aprendizaje.

\section{Instrumentos}

Para evaluar las habilidades metalingüísticas, la lectura y el funcionamiento cognitivo se utilizaron escalas de la batería de Evaluación Neuropsicológica Infantil - ENI (MATUTE; ROSELLI; ARDILA; OSTROVSKY-SOLÍS, 2007).

\section{Habilidades Metalingüisticas}

Síntesis fonémica. Evalúa la capacidad del niño para formar palabras al escuchar los fonemas que la integran. Se le dicen los sonidos constitutivos de una palabra (p. ej., / k /, / a /, / s /, / a /) y el niño debe decir la palabra. Se presentan ocho palabras.

Deletreo. Se pide deletrear ocho palabras.

Conteo de sonidos. Se pide que cuente los sonidos que integran cada una de las ocho palabras.

Conteo de palabras. Debe decir el número de palabras que hay en una oración después de que se le lea. Se presentan ocho oraciones.

\section{Lectura}

Lectura de sílabas. El niño debe leer ocho sílabas.

Lectura de palabras. El niño debe leer ocho palabras.

Lectura de no-palabras. El niño debe leer ocho palabras sin sentido. 
Lectura de oraciones Aciertos. Ante la lámina utilizada en seguimiento de instrucciones, el niño debe leer en voz alta 10 oraciones que incluyen instrucciones (ej., "Señala un avión grande.").

Lectura de oraciones Comprensión. Si el niño realiza la instrucción de manera correcta inmediatamente después de haberse leído.

\section{Atención visual}

Cancelación de dibujos. Incluye una página con una serie de dibujos de 44 conejos grandes y pequeños. El niño debe tachar con un lápiz los conejos grandes, lo más rápido posible, dentro de un tiempo límite de un minuto.

Cancelación de letras (paradigma AX). Incluye una página con 82 letras distribuidas en varios renglones. El niño debe tachar con un lápiz la letra $\mathrm{X}$, únicamente cuando ésta está precedida por la letra A. El tiempo límite es un minuto.

\section{Atención auditiva y memoria de trabajo}

Dígitos en progresión. El niño debe repetir series de números, empezando por series de dos números y terminando con una serie de ocho números.

Dígitos en regresión. El niño debe repetir en orden inverso series de números, comenzando con series de dos dígitos y terminando con series de 7 .

\section{Funciones ejecutivas}

Fluidez verbal Semántica. Incluye dos categorías: animales y frutas. Se aplican de manera individual. El niño debe decir el mayor número posible de animales (o frutas) en un minuto.

Fluidez verbal Fonémica. Número total de palabras producidas en un minuto que comiencen con la letra $\mathrm{M}$.

Flexibilidad cognitiva. Clasificación de tarjetas. El niño tiene que decidir cuál es el principio (color, forma o número) que subyace a la agrupación de tarjetas, con la retroalimentación (correcto o incorrecto), que da el examinador a sus respuestas. Se califica el número de errores, el número de respuestas correctas y el número de categorías.

Planeación y organización. La pirámide de México. Se utilizan tres bloques de tres colores diferentes (verde, blanco y rojo) y tamaños (grande, mediano y pequeño). En tarjetas se presentan una a una diversas formas de construcción 
con los bloques. El niño tiene que hacer con los bloques la construcción que se le pide cada vez, empleando el menor número de movimientos posibles de los bloques y siguiendo las instrucciones específicas a la tarea.

\section{Razonamiento}

Similitudes. Se presentan ocho pares de palabras, y cada pareja pertenece a una misma categoría semántica (gato y perro). El niño tiene que identificar al menos un atributo común a los dos conceptos.

Matrices. Se presentan ocho figuras que tienen una parte que falta. El niño tiene que decidir cuál es la parte que le falta a cada una de las figuras, y escoger entre las posibilidades que se le presentan.

Problemas numéricos. El niño responde verbalmente a ocho problemas aritméticos que se presentan en tarjetas.

\section{Memoria}

Lista de palabras. Se presentan, en cuatro ensayos consecutivos, nueve palabras (para los niños de 5-8 años) o 12 (para los niños de 9-16 años). Las palabras pertenecen a tres categorías semánticas: animales, frutas y partes del cuerpo. El niño debe recordar las palabras leídas. Se computa la recuperación espontánea de la lista de palabras (Evocación libre de las palabras presentadas previamente) y se calcula como la suma del total de palabras recuperadas en los cuatro ensayos.

Recuperación por claves. Se le indica al niño cada una de las categorías (frutas, animales y partes del cuerpo) en las que se incluyen las palabras presentadas anteriormente y el niño tiene que decir las palabras pertenecientes a cada una de ellas.

Reconocimiento verbal-auditivo. En una lista de 18 palabras para los niños de 5-8 años y de 24 para los niños de 9-16 años de edad, el niño debe reconocer las palabras presentadas en la prueba de Lista de palabras.

\section{Procedimiento}

Se solicitó el consentimiento informado a los padres o cuidadores de los niños, en el cual se explicaba detalladamente en que consistía la evaluación. 
Todas las pruebas fueron administradas por profesionales y alumnos avanzados de la carrera de Psicología, especialmente entrenados para la tarea, en aulas de las instituciones educativas, en forma individual y en dos tomas consecutivas de una duración aproximada de 30 minutos.

\section{Resultados}

A continuación se presentan las medias y los desvíos estándares de todas las variables analizadas en el estudio y a fines de observar su comportamiento se incluyen los estadísticos descriptivos de las muestras normativas para niños y niñas de esa edad, de la Batería de Evaluación Neuropsicológica Infantil ENI (MATUTE et al., 2007).

TABLA 1 - ESTADÍSTICOS DESCRIPTIVOS PARA TODA LA MUESTRA Y DATOS NORMATIVOS DE LA ENI

continua

\begin{tabular}{|c|c|c|c|c|}
\hline \multirow[t]{2}{*}{ Proceso } & \multirow{2}{*}{ Variable } & \multicolumn{2}{|c|}{ Estudio } & \multirow{2}{*}{$\begin{array}{c}\begin{array}{c}\text { Normativas } \\
\text { ENI }\end{array} \\
\text { Media }\end{array}$} \\
\hline & & Media & DE & \\
\hline \multirow{4}{*}{$\begin{array}{c}\text { Habilidades } \\
\text { Metalingüísticas }\end{array}$} & Síntesis fonológica & 1,60 & 1,74 & 1,5 \\
\hline & Conteo de sonidos & 3,53 & 2,68 & 2 \\
\hline & Deletreo & 2,95 & 2,41 & 2 \\
\hline & Conteo de palabras & 2,08 & 2,28 & 1 \\
\hline \multirow{4}{*}{ Lectura } & Lectura de palabras & 5,45 & 4,47 & 5,5 \\
\hline & Lectura de no-palabras & 3,67 & 3,49 & 4 \\
\hline & $\begin{array}{l}\text { Lectura de oraciones } \\
\text { Precisión }\end{array}$ & 4,54 & 4,45 & 4 \\
\hline & $\begin{array}{l}\text { Lectura de oraciones } \\
\text { Comprensión }\end{array}$ & 3,24 & 3,42 & 3 \\
\hline Atención visual & Cancelación dibujos & 12,38 & 5,29 & 10,5 \\
\hline
\end{tabular}


TABLA 1 - ESTADÍSTICOS DESCRIPTIVOS PARA TODA LA MUESTRA Y DATOS NORMATIVOS DE LA ENI

\begin{tabular}{|c|c|c|c|c|}
\hline \multirow[t]{2}{*}{ Proceso } & \multirow{2}{*}{ Variable } & \multicolumn{2}{|c|}{ Estudio } & \multirow{2}{*}{$\begin{array}{c}\text { Normativas } \\
\text { ENI } \\
\text { Media }\end{array}$} \\
\hline & & Media & DE & \\
\hline & Cancelación letras & 12,80 & 4,84 & 8,5 \\
\hline \multirow[t]{2}{*}{$\begin{array}{c}\text { Atención } \\
\text { auditiva y } \\
\text { memoria de } \\
\text { trabajo }\end{array}$} & Dígitos en progresión & 4,42 & 1,01 & 3 \\
\hline & Dígitos en regresión & 2,39 & 0,98 & 1 \\
\hline \multirow{7}{*}{$\begin{array}{l}\text { Funciones } \\
\text { ejecutivas }\end{array}$} & Fluidez semántica Frutas & 6,07 & 1,94 & 7 \\
\hline & $\begin{array}{l}\text { Fluidez semántica } \\
\text { Animales }\end{array}$ & 8,27 & 3,32 & 10 \\
\hline & Fluidez fonológica & 3,72 & 2,39 & 1,5 \\
\hline & Flexibilidad \% Errores & 35,39 & 13,99 & 46 \\
\hline & $\begin{array}{l}\text { Flexibilidad \% } \\
\text { Perseveraciones }\end{array}$ & 23,24 & 16,60 & 41,5 \\
\hline & $\begin{array}{l}\text { Pirámide México diseños } \\
\text { correctos }\end{array}$ & 8,32 & 2,62 & 7,5 \\
\hline & $\begin{array}{l}\text { Pirámide México mínimos } \\
\text { movimientos }\end{array}$ & 5,52 & 2,64 & 5 \\
\hline \multirow[t]{3}{*}{ Razonamiento } & Similitudes & 3,80 & 2,39 & 3 \\
\hline & Matrices & 1,38 & 1,61 & 1 \\
\hline & Problemas numéricos & 1,85 & 0,98 & 1 \\
\hline \multirow{4}{*}{ Memoria } & Memoria Inmediata Total & 19,33 & 4,46 & 20 \\
\hline & Recuperación MLP & 4,72 & 2,13 & 5 \\
\hline & Recuperación con claves & 4,78 & 1,92 & 5 \\
\hline & Reconocimiento & 15,21 & 2,37 & 16 \\
\hline
\end{tabular}


Los resultados permiten observar que el desempeño de los niños evaluados se asemeja en casi todos los casos a los valores normativos de la ENI en lectura, en memoria y en razonamiento, se destacan diferencias de más de un $50 \%$ a favor de los niños de la muestra en las habilidades metalingüísticas y diferencias menores en atención visual y auditiva, fluidez fonológica y menores porcentajes de errores y perseveraciones en pruebas de flexibilidad. Debemos destacar que los datos normativos se obtuvieron con población hispanoparlante de México y Colombia.

Con el objeto de explorar la presencia y los niveles de asociación entre el desempeño en lectura, el funcionamiento cognitivo y las habilidades metalingüísticas, se realizó un análisis de correlaciones bivariadas. A continuación, en la Tabla 2 se exponen los resultados.

TABLA 2 - CORRELACIONES BIVARIADAS ENTRE LA LECTURA Y LAS VARIABLES DEL ESTUDIO

continua

\begin{tabular}{|c|c|c|c|c|c|}
\hline Proceso & & $\begin{array}{l}\text { Lectura de } \\
\text { palabras }\end{array}$ & $\begin{array}{c}\text { Lectura de } \\
\text { no-palabras }\end{array}$ & $\begin{array}{c}\text { Lectura } \\
\text { oraciones } \\
\text { Precisión }\end{array}$ & $\begin{array}{c}\text { Lectura } \\
\text { oraciones } \\
\text { Comprensión }\end{array}$ \\
\hline \multirow{4}{*}{$\begin{array}{c}\text { Habilidades } \\
\text { Metalingüísticas }\end{array}$} & Síntesis fonológica &, $547(* *)$ &, $561(* *)$ &, $527(* *)$ &, $492(* *)$ \\
\hline & Conteo de sonidos &, $804(* *)$ &, $777(* *)$ &, $770(* *)$ &, $712(* *)$ \\
\hline & Deletreo &, $818(* *)$ &, $825(* *)$ &, $778(* *)$ &, $749(* *)$ \\
\hline & Conteo palabras &, $597(* *)$ &, $601(* *)$ &, $578(* *)$ &, $614(* *)$ \\
\hline \multirow[t]{2}{*}{$\begin{array}{c}\text { Atención } \\
\text { visual }\end{array}$} & Cancelación dibujos &, $332(* *)$ &, $327(* *)$ &, $298(* *)$ &, $295(* *)$ \\
\hline & Cancelación letras &, $285(* *)$ & ,298(**) &, $266(* *)$ &, $250(* *)$ \\
\hline \multirow{2}{*}{$\begin{array}{c}\text { Atención } \\
\text { auditiva y } \\
\text { memoria de } \\
\text { trabajo }\end{array}$} & $\begin{array}{l}\text { Dígitos en } \\
\text { progresión }\end{array}$ &, $508(* *)$ &, $504(* *)$ &, $507(* *)$ &, $546(* *)$ \\
\hline & Dígitos en regresión &, $549(* *)$ &, $536(* *)$ &, $512(* *)$ &, $513(* *)$ \\
\hline
\end{tabular}


TABLA 2 - CORRELACIONES BIVARIADAS ENTRE LA LECTURA Y LAS VARIABLES DEL ESTUDIO

conclusión

\begin{tabular}{|c|c|c|c|c|c|}
\hline Proceso & & $\begin{array}{l}\text { Lectura de } \\
\text { palabras }\end{array}$ & $\begin{array}{c}\text { Lectura de } \\
\text { no-palabras }\end{array}$ & $\begin{array}{c}\text { Lectura } \\
\text { oraciones } \\
\text { Precisión }\end{array}$ & $\begin{array}{c}\text { Lectura } \\
\text { oraciones } \\
\text { Comprensión }\end{array}$ \\
\hline \multirow{7}{*}{$\begin{array}{l}\text { Funciones } \\
\text { ejecutivas }\end{array}$} & $\begin{array}{l}\text { Fluidez semántica } \\
\text { Frutas }\end{array}$ &, 144 &, 144 &, $174(*)$ & ,198(*) \\
\hline & $\begin{array}{l}\text { Fluidez semántica } \\
\text { Animales }\end{array}$ &, $360(* *)$ &, $377(* *)$ &, $348(* *)$ &, $402(* *)$ \\
\hline & Fluidez fonológica &, $560(* *)$ &, $569(* *)$ &, $557(* *)$ &, $528(* *)$ \\
\hline & $\begin{array}{l}\text { Flexibilidad \% } \\
\text { Errores }\end{array}$ &,$- 281(* *)$ &,$- 278(* *)$ &,$- 217(* *)$ &,$- 195(*)$ \\
\hline & $\begin{array}{l}\text { Flexibilidad \% } \\
\text { Perseveraciones }\end{array}$ &,$- 301(* *)$ &,$- 323(* *)$ &,$- 223(* *)$ &,$- 239(* *)$ \\
\hline & $\begin{array}{l}\text { Pirámide México } \\
\text { correctos }\end{array}$ &, $213(* *)$ &, $161(*)$ & ,209(*) &, $159(*)$ \\
\hline & $\begin{array}{l}\text { Pirámide México } \\
\text { mínimo movimientos }\end{array}$ & ,199(*) &, 144 &, $179(*)$ & ,094 \\
\hline \multirow{3}{*}{ Razonamiento } & Similitudes &, $232(* *)$ &, $272(* *)$ &, $252(* *)$ &, $230(* *)$ \\
\hline & Matrices &, $152(*)$ &, 116 &, 124 &, 119 \\
\hline & Problemas numéricos &, $527(* *)$ &, $507(* *)$ &, $497(* *)$ &, $490(* *)$ \\
\hline \multirow{4}{*}{ Memoria } & $\begin{array}{l}\text { Memoria Inmediata } \\
\text { Total }\end{array}$ &, $149(*)$ & ,095 &, 074 &, 072 \\
\hline & Recuperación MLP &, $224(* *)$ &, $216(* *)$ &, 132 &, $159(*)$ \\
\hline & $\begin{array}{l}\text { Recuperación con } \\
\text { claves }\end{array}$ &, $328(* *)$ &, $338(* *)$ &, $255(* *)$ &, $266(* *)$ \\
\hline & Reconocimiento &, 136 &, 111 & ,088 & ,088 \\
\hline
\end{tabular}

* La correlación es significativa al nivel 0,05 .

** La correlación es significante al nivel 0,01 .

Se destacan en color gris las correlaciones estadísticamente significativas.

La tabla permite confirmar la existencia de asociaciones estadísticamente significativas de todas las variables que evalúan la lectura (palabras - no-palabras - oraciones precisión y comprensión) con prácticamente todas las funciones 
cognitivas y las habilidades metalingüísticas. Salvo el reconocimiento auditivo, que representa la habilidad de incorporación de información en la memoria de largo plazo (aprendizaje), la memoria inmediata total, que también representa la capacidad de aprendizaje de palabras luego de cuatro ensayos, el resto de funciones se asocian al aprendizaje de la lectura. Es destacable que las asociaciones de mayor peso se dan con las habilidades metalingüísticas (entre 0,492 y 0,825 con niveles de significación menores a 0,001 ).

\section{Discusión de los resultados}

En principio, debemos destacar que los resultados aportan evidencia empírica de las relaciones que tiene el funcionamiento cognitivo con el aprendizaje de la lectura.

El desempeño en las tareas que evalúan habilidades metalingüísticas se asocia, de forma sistemática y sólida, con niveles medios y altos a todas las tareas de precisión de lectura evaluadas, de palabras, de no-palabras y de oraciones y de comprensión lectora, demostrando la importancia de su dominio como prerrequisito para favorecer la alfabetización. Aquellos niños y niñas que demuestran mejores desempeños en conciencia fonológica, conteo de sonidos de letras y palabras y deletreo tienden, de forma sistemática, a presentar mejores desempeños en lectura. El conocimiento y la reflexión consciente sobre los diferentes niveles del sistema lingüístico parecen tener vital importancia para la adquisición de la lectura. La toma de conciencia sobre los sonidos de la propia lengua (conciencia fonológica) y la capacidad de aislar las palabras que componen una oración y comprender que se trata de una unidad lingüística separada (conciencia léxica) se van perfeccionando, alcanzando grados mayores de experticia con el aprendizaje de la lectura, sin embargo parecen ser claves para el proceso de alfabetización. Esos resultados confirman los supuestos de estudios anteriores (BORZONE et al., 2004; BALL, 1997; OTT, 1997; STILLER, 2005) y permiten sostener que ambos se apoyan mutuamente entre si y mejoran con la escolarización. Sin embargo, coincidimos con Guimarães (2002) e insistimos en que su desarrollo es un tema tan complejo que requiere que se realicen estudios longitudinales que permitan determinar cómo se relacionan con la lectura durante todo el proceso de adquisición.

También los resultados permiten confirmar la importancia de la ruta sublexical o fonológica para el aprendizaje de la lectura. 
Con respecto a la relación con los procesos cognitivos, los resultados reafirman la idea de que la adquisición de la lectura es un proceso complejo que descansa en el desarrollo de diversas funciones y habilidades mentales (GOUGH, 2002; GOUGH; COSKY, 1975; JUST; CARPENTER; WOOLEY, 1982). Evidentemente, los datos obtenidos permiten confirmar que la adquisición de la lectura se entiende como un proceso que tiene prerrequisitos cognitivos. La memoria, necesaria para aprender, es decir, codificar, almacenar y recuperar información, es fundamental en la lectura, básicamente para la adquisición de correspondencias grafemas-fonemas, para el vocabulario y para las reglas que guían la escritura en el español.

Confirmando lo expuesto por Rosselli et al. (2006), hemos demostrado que la lectura exige una serie de habilidades de tipo cognitivo como la atención, la memoria, el funcionamiento ejecutivo y la atención, para lograr una adecuada decodificación de los estímulos y comprensión del texto. Si bien los predictores cognitivos de la lectura varían (SAVAGE et al., 2005, CANETJURIC; URQUIJO; RICHARD'S; BURIN, 2009, ALTEMEIER et al., 2006; WILLCUTT et al., 2005; SWANSON, 1999), no quedan dudas de su importancia en la adquisición de la lectura, al menos en el primer año. Probablemente estas relaciones tiendan a disminuir en función del ejercicio, el aprendizaje y el desarrollo de los procesos cognitivos.

De ninguna manera debemos perder de vista la idea de que además de los factores cognitivos individuales, el aprendizaje de la lectura se asocia a otras variables de importancia, como la exposición a la lectura, al contexto alfabetizador, o al desarrollo de una actitud y motivación positivas. Leer y escribir constituyen esencialmente prácticas sociales y comunicativas.

Finalmente, si bien este estudio, de carácter transversal, no permite captar la influencia del desarrollo cognitivo para explicar la adquisición de la lectura, aporta evidencias de que su afectación provocaría una disfunción con efectos sobre los procesos de aprendizaje formal académico.

\section{REFERENCIAS}

AKSAN, N.; KOCHANSKA, D. Links between systems of inhibition from infancy to preschool years. Child Development, n. 75, p. 1477-90, 2004.

ALTEMEIER, L.; JONES, J.; ABBOTT, R. D.; BERNINGER, V. W. Executive Functions in Becoming Writing Readers and Reading Writers: Note Taking and 
Report Writing in Third and Fifth. Developmental Neuropsychology, v. 29, n. 1, p. 161-173, 2006.

ANDERSON, P.; DOYLE, L. Neurobiological outcomes of school-age children born extremely low birth weight or very preterm. Journal American Medical Association, n. 289, p. 3264-72, 2003.

ANDERSON, V. Assessing executive functions in children: Biological, psychological, and developmental considerations. Developmental Neurorehabilitation, n. 4, p. 119-136, 2001.

ANDRÉS, M. L.; URQUIJO, S.; NAVARRO, J. I.; GARCÍA-SEDEÑO, M. Contexto alfabetizador familiar: relaciones con la adquisición de habilidades prelectoras y desempeño lector. European Journal of Education and Psychology, v. 3, n. 1, p. 129-140, 2010.

ARDILA, A. Semantic paralexias in spanish language. Aphasiology, n. 12, p. 885-900, 1998.

BADDELEY, A. D. The central executive: A concept and some misconceptions. Journal of the International Neuropsychological Society, n. 4, p. 523-526, 1998.

. Exploring the central executive. The Quarterly Journal of Experimental Psychology, n. 49A, p. 5-28, 1996.

. Working Memory. Science, n. 255, p. 556-559, 1992.

BADDELEY, A. D.; DELLA SALA, S. Working memory and executive control. Philosophical Transactions of the Royal Society of London, n. 351, p. 13971404, 1996.

BADDELEY, A. D.; HITCH, G. J. Developments in the Concept of Working Memory. Neuropsychology, n. 8, p. 485-493, 1994.

BADDELEY, A.; LARSEN, J. The phonological loop: Some answers and some questions. Quarterly Journal of Experimental Psychology, v. 60, n. 4, p. 512-518, 2007.

BALL, E. W. Phonological awareness: Implications for whole language and emergent literacy programs. Topics in Language Disorders, n. 17, p. 14-26, 1997. 
BORZONE, A. M.; ROSEMBERG, C.; DIUK, B.; SILVESTRI, A.; PLANA, D. Niños y Maestros por el camino de la Alfabetización. Programa Infancia y Desarrollo. Buenos Aires: Ministerio de Educación, Ciencia y Tecnología de la Nación, 2004.

CANET-JURIC, L.; URQUIJO, S.; RICHARD'S, M. M.; BURIN, D. Predictores cognitivos de niveles de comprensión lectora mediante análisis discriminante. International Journal of Psychological Research, v. 2, n. 2, p. 99-111, 2009.

CATHERWOOD, D. New views on the young brain: offerings from developmental psychology to early childhood education. Contemporary Issues Early Childhood, n. 1, p. 23-35, 2000.

CHÁVEZZAMORA, J. M. Guía para el desarrollo de los Procesos Metacognitivos. Perú: Kinko’s Impresores, 2006.

COLLETTE, F.; HOGGE, M.; SALMON, E.; VAN DER LINDEN, M. Exploration of the neural substrates of executive functioning by functional neuroimaging. Neuroscience, n. 139, p. 209-221, 2006.

COLTHEART, M. Disorders of reading and their implications for models of normal reading. Visible Language, n. 15, p. 245-86, 1981.

DEAK, G.; NARASIMHAM, G. Is perseveration caused by inhibition failure? Evidence from preschool children's inferences about word meanings. $J$ Exp Psychol, n. 86, p. 194-222, 2003.

DEFIOR CITOLER, S. A. La conciencia fonológica y la adquisición de la lectoescritura. Infancia y Aprendizaje, n. 67-68, p. 91-114, 1994.

DELIS, D.; KAPLAN, E.; KRAMER, N. Delis-Kaplan Executive Function System. Odessa, FL: Psychological Assessment Resources, Inc., 2001.

DELUCA, C.; WOOD, S. J.; ANDERSON, V.; BUCHANAN, J. A.; PROFFITT, T.; MAHONY, K. et al. Normative data from CANTAB: Development of executive function over the lifespan. Journal of Clinical and Experimental Neuropsychology, n. 25, p. 242-254, 2003.

DENCKLA, M. B. Measurement of executive function. In: LYON, G. R. (Ed.). Frames of reference for the assessment of learning disabilities: new views on measurement issues. Baltimore: Brookes, 1994. p. 117-142. 
DIAMOND, A. Normal development of prefrontal cortex from birth to young adulthood: Cognitive functions, anatomy, and biochemistry. In: STUSS, D. T.; KNIGHT, R. T. (Eds.). Principles of frontal lobe function. London, UK: Oxford University Press, 2002. p. 466-503.

ELLIOTT, R. Executive functions and their disorders. British Medical Bulletin, n. 65 , p. $49-59,2003$.

ETCHEPAREBORDA, M. C; ABAD-MAS, L. Memoria de trabajo en los procesos básicos del aprendizaje. Revista de NeurologíaNeurologia, n. 40 (Supl 1), p. S79-83, 2005.

FEINSTEIN, L.; BYNNER, J. The importance of cognitive development in middle childhood for adult socioeconomic status, mental health and problem behavior. Child Development, n. 75, p. 1329-39, 2004.

GEE, J. Critical issues: Reading and de new literacy studies. Reframing the National Academy of Science Report on Reading. Journal of Literacy Research, n. 31, p. 355-374, 1999.

GOLDMAN-RAKIC, P. S. The prefrontal landscape: Implications of functional architecture for understanding human mentation and the central executive. Philosophical Transactions of the Royal Society of London. Series B. Biological Sciences, n. 35, p. 1445-1454, 1996.

GOSWAMI, U. Cognition in children. East Sussex: Psychology Press, 1998.

GOUGH, P. B. Word recognition. In: PEARSON, P. D. (Ed.). Handbook of reading research. Mahwah, NJ: Lawrence Erlbaum, 2002. p. 225-54.

GOUGH, P. B.; COSKY, M. J. One second of reading again. In: CASTELLAN, J.; PISONI, G. (Eds.). Cognitive theory. Hillsdale, NJ: Erlbaum, 1975. v. 2. p. 271-88.

GUIMARÃES, S. R. K. Dificuldades no Desenvolvimento da Lectoescrita: O Papel das Habilidades Metalingüísticas. Psicología: Teoria e Pesquisa, n. 18, p. 247-259, 2002.

HAAN, M.; JOHNSON, M. H. (Eds.). The cognitive neuroscience of development. Hove: Psychology Press, 2003. 
HOURIGAN, M. Literacy as social exchange: Intersections of class, gender, and culture. New York: State University of New York, 1994.

JUST, M. A.; CARPENTER, P. A.; WOOLEY, J. D. Paradigms and processes in reading comprehension. Psychol Rev, n. 87, p. 329-54, 1982.

LAFLECHE, G.; ALBERT, M. Executive function deficits in mild Alzheimer's disease. Neuropsychology, n. 9, p. 313-320, 1995.

LEE, C. A Culturally based cognitive apprenticeship: Teaching African American high School students skills in literary interpretation. Reading Research Quarterly, n. 30, p. 608-630, 1995.

LEE, K.; NG, S. F.; NG, E. L.; LIM, Z. Y. Working memory and literacy as predictors of performance on algebraic word problems. Journal of Experimental Child Psychology, v. 89, n. 2, p. 140-158, 2004.

LUCIANA, M.; CONKLIN, H. M.; HOOPER, C. J.; YARGER, R. S. The development of nonverbal working memory and executive control processes in adolescents. Child Development, n. 76, p. 697-712, 2005.

LUCIANA, M.; NELSON, C. A. Assessment of neuropsychological function in children using the Cambridge Neuropsychological Testing Automated Battery (CANTAB): Performance in 4 to 12 year-olds. Developmental Neuropsychology, n. 22, p. 595-623, 2002.

LUNA, B.; GARVER, K. E.; URBAN, T. A.; LAZAR, N. A.; SWEENEY, J. A. Maturation of cognitive processes from late childhood to adulthood. Child Development, n. 75, p. 1357-1372, 2004.

LYONS-WARREN, A.; LILLIE, R.; HERSHEY, T. Short and long-term spatial delayed response performance across the lifespan. Developmental Neuropsychology, n. 26, p. 661-678, 2004.

MATUTE, E.; LEAL, F. Los llamados errores ortográficos en niños hispanohablantes con problemas en el aprendizaje de la lectoescritura. In: MATUTE, E.; LEAL, F. (Eds.). Introducción al estudio del español desde una perspectiva multidisciplinaria. Guadalajara: Universidad de Guadalajara, 2003. p. 549-70.

MATUTE, E.; ROSSELLI, M.; ARDILA, A.; OSTROSKY-SOLÍS, F. Evaluación Neuropsicológica Infantil (ENI), 2007. Manual. 
MEHLER, J.; DUPOUX, D. Nacer sabiendo. Madrid: Alianza, 1992.

MELLIER, D.; FESSARD, C. Preterm birth and cognitive inhibition. Eur Rev Appl Psychol, n. 48, p. 13-18, 1998.

MOJE, E. B.; DILLON, D. R.; O'BRIEN, D. G. Re-examining the roles of learner, the text, and the context in secondary literacy. Journal of Educational Research, n. 93, p. 165-180, 2000.

MORASO, M. C.; DURO, E. Nutrición, desarrollo y alfabetización. Buenos Aires: UNICEF, 2004. Disponible en: <http://www.unicef.org/argentina/spanish/ ar_insumos_NAD2.pdf $>$. Consultado en: 24/9/2007.

MUNOZ, D.; BROUGHTON, J.; GOLDRING, J.; ARMSTRONG, I. Age-related performance of human subjects on saccadic eye movement tasks. Experimental Brain Research, n. 217, p. 1-10, 1998.

OTT, P. How to detect and manage dyslexia - A reference and resource manual. London: Heinemann Educational Pub., 1997.

PAPAZIAN, O.; ALFONSO, I.; LUZONDO, R. J. Trastornos de las funciones ejecutivas. Revista de Neurología, n. 42 (Supl. 3), p. S45-S50, 2006.

PENNINGTON, B. F.; OZONOFF, S. Executive Functions and Developmental Psychopatology. Journal of Child Psychology and Psychiatry, n. 37, p. 51-87, 1996.

PIGUET, O.; GRAYSON, G.; BROWE, A.; TATE, H.; LYE, T.; CREASEY, H. et al. Normal Aging and Executive Functions in "Old-old" Community Dwellers: Poor performance is not an inevitable outcome. International Psychogeriatric Association, n. 14, p. 139-159, 2002.

PURVIS, K. L.; TANNOCK, R. Language Abilities in Children with Attention Deficit Hyperactivity Disorder, Reading Disabilities, and Normal Controls. Journal of Abnormal Child Psychology, v. 25, n. 2, p. 133-144, 1997.

ROBERTS, R. J.; PENNINGTON, B. F. An interactive framework for examining prefrontal cognitive processes. Developmental Neuropsychology, n. 12, p. 105-126, 1996.

ROSSELLI, M.; MATUTE, E.; ARDILA, A. Predictores neuropsicológicos de la lectura en español. Revista de Neurología, n. 42, p. 202-10, 2006. 
RUMSEY, R. K. Executive functioning in boys and girls with attention-deficit/ hyperactivity disorder with and without a comorbid reading disability. The Sciences and Engineering, v. 64, n. 11-B, p. 5820, 2004.

SASTRE-RIBA, S. Condiciones tempranas del desarrollo y el aprendizaje: el papel de las funciones ejecutivas. Rev. Neurol., n. 42 (Supl 2), p. S143-S151, 2006.

SAVAGE, R. S.; FREDERICKSON, N.; GOODWIN, R.; PATNI, U.; SMITH, N.; TUERSLEY, L. Relationship among rapid digit naming, phonological processing, motor automaticity, and speech perception in poor, average, and good readers and spellers. J Learn Disab, n. 38, p. 12-28, 2005.

SCRIBNER, S.; COLE, M. The psychology of literacy. Cambridge, M.A.: Harvard University Press, 1981.

SENGSTOCK, S. K. The contribution of working memory and inhibition to the executive functioning of children with attention deficit hyperactivity disorder and children with reading disability. The Sciences and Engineering, v. 61, n. 11-B, p. 6148, 2001.

SHALLICE, T. From Neuropsychology to mental structure. Cambridge: Cambridge University Press, 2001.

SOMMERFELT, K.; ANDERSSON, H. W.; SONNANDER, K.; AHLSTEN, G.; ELLERTSEN, B.; MARKESTAD, T. et al. Cognitive development of term small for gestational age children at five years age. Arch Disabl Child, n. 83, p. 25-30, 2000.

STILLER, P. A. Effectiveness of intensive phonemic awareness intervention on upper elementary students with learning disabilities. Unpublished master's thesis, California State University, California, 2005.

STREET, B. Literacy in theory and practice. London: Cambridge University Press, 1984.

STRINGER, R. W.; TOPLAK, M. E.; STANOVICH, K. E. Differential Relationships between RAN Performance, Behaviour Ratings, and Executive Function Measures: Searching for a Double Dissociation. Reading and Writing: An Interdisciplinary Journal, v. 17, n. 9, p. 891-914, 2004. 
SWANSON, H. L. Reading Comprehension and Working Memory in LearningDisabled Readers: Is the Phonological Loop More Important Than the Executive System? Journal of Experimental Child Psychology, v. 72, n. 1, p. 31, 1999.

URQUIJO, S. Aprendizaje de la lectura. Diferencias entre escuelas de gestión pública y de gestión privada. Revista Evaluar, n. 9, p. 19-34, 2009.

WIGFIELD, A.; ASHER, S. R. Social and motivational influences on reading. In: PEARSON, P. D. (Ed.). Handbook of reading research. Mahwah, NJ: Lawrence Erlbaum, 2002. p. 423-52.

WILLCUTT, E. G.; PENNINGTON, B. F.; OLSON, R. K.; CHHABILDAS, N.; HULSLANDER, J. Neuropsychological Analyses of Comorbidity Between Reading Disability and Attention Deficit Hyperactivity Disorder: In Search of the Common Deficit. Developmental Neuropsychology, v. 27, n. 1, p. 35-78, 2005 .

WODKA, E.; MAHONE, M.; BLANKNER, J.; LARSON, J.; FOTEDAR, S.; DENCKLA, M.; MOSTOFSKY, S. Evidence that response inhibition is a primary déficit in ADHD. Journal of Clinical and Experimental Neuropsychology, $\mathrm{n}$. 29, p. 345-356, 2007.

ZELAZO, P. D.; CRAIK, F. I. M.; BOOTH, L. Executive function across the life span. Acta Psychologica, n. 115, p. 167-183, 2004.

ZELAZO, P. H. The development of conscious control in childhood. Trends Cogn Sci, n. 8, p. 12-7, 2004.

Texto recebido em 14 de junho de 2010 .

Texto aprovado em 5 de julho de 2010. 